\title{
Magnetic resonance imaging and neurological recovery in acute spinal cord injury: observations from the National Acute Spinal Cord Injury Study 3
}

\author{
MJ Shepard ${ }^{1}$ and MB Bracken*,1,2 \\ ${ }^{1}$ Department of Epidemiology and Public Health, Yale University School of Medicine, New Haven, Connecticut, \\ USA; ${ }^{2}$ Department of Neurology, Yale University School of Medicine, New Haven, Connecticut, USA
}

\begin{abstract}
Study design: Data are from a multicenter, randomized, double blind clinical trial of acute spinal cord injury.

Objectives: To evaluate the prognostic value of magnetic resonance imaging (MRI) for randomized patients in the National Acute Spinal Cord Injury Study 3 (NASCIS).

Setting: Sixteen spinal cord injury centers throughout the United States and Canada.

Methods: Of 499 patients randomized in NASCIS 3 between December 1991 and September 1995, MRI was electively done on 191 patients within $72 \mathrm{~h}$ of injury. Indications of hemorrhage, edema, and contusion were recorded by standard protocol. Neurological impairment as determined by motor function, response to pin prick and light touch was assessed at admission to the participating center and 6 weeks after injury. Change in neurological function was obtained by subtracting the score of each neurological parameter at admission from that measured at 6 weeks. Spinal cord surgery performed within the 3 days after injury was noted. Data were analyzed by: chi square, analysis of variance, multiple logistic regression and linear regression models.

Results: Patients with hemorrhage were much more likely to have a complete injury $(\mathrm{OR}=2.88,95 \mathrm{Cl} 1.32,6.23)$; however this association was much reduced when the initial neurological examination was taken into account $(\mathrm{AOR}=1.43,95 \% \mathrm{Cl} 0.55,3.73)$ and was no longer a significant predictor of injury. MRI evidence of cord edema was the strongest predictor of reduced improvement in motor function $(-3.34$ points, $P=0.06)$ and light touch sensation $(-3.41$ points, $P=0.05)$ at 6 weeks.

Conclusions: Cord hemorrhage, contusion, and edema on MRI were not associated with diagnosis of a complete cord injury after neurological assessment from the initial clinical examination was taken into account. Prediction of a worse 6 week neurological status was weakly associated with the presence of edema diagnosed by MRI. As MRI technology improves, these diagnostic and predictive capabilities need to be re-assessed.

Sponsorship: NASCIS 3 was funded by the National Institute of Neurological Disorders and Stroke at the National Institutes of Health, Washington, DC, USA. Pharmacia and Upjohn provided study drugs and placebos; they also monitored data quality, and funded additional tests, in accordance with Food and Drug Administration regulatory requirements. Dr Bracken has served as an occasional paid consultant to Pharmacia and Upjohn.
\end{abstract}

Keywords: magnetic resonance imaging; prognostic ability; neurological function; spinal cord injury

\section{Introduction}

Not until the 1980s did magnetic resonance imaging (MRI) become part of the diagnostic armamentaria for spinal cord injury (SCI). A number of factors interfered with earlier use of MRI for these injuries, such as slow scanning speeds, low spatial resolution,

*Correspondence: Dr MB Bracken, Department of Epidemiology and Public Health, Yale University School of Medicine, Box 208034, 60 College Street, New Haven, CT 06520-8034, USA and the difficulty of stabilizing patients during lengthy scans. In addition the magnetic fields of MRI precluded the use of ferrous stabilization equipment. ${ }^{1}$ Studies published in the early to mid 1990s established the diagnostic value of MRI in spinal cord injury, with its enhanced ability to depict hemorrhage, cord edema, contusion, and stenosis, as well as injury to vertebral bodies and paraspinal tissues. ${ }^{2-4}$ However, the prognostic value of MR imaging in spinal cord injury is less clear. ${ }^{5-7}$ 
At the time of the National Acute Spinal Cord Injury Study 2 (NASCIS 2, 1985-89) ${ }^{8}$ few centers used MR imaging to assess spinal cord injury. During this time, level and degree of injury were determined primarily through plain spine X-rays, myelogram, or CT scan. However, refinement of MR imaging scanners, improvements in reading MR imaging, and the development of non-ferrous materials for patient stabilization resulted in its increased use in assessing SCI. By the time NASCIS 3 randomized its first patient in December 1991, MR imaging was a frequently used diagnostic tool in a number of spinal cord injury centers. Because of this, an MR imaging protocol to evaluate spinal cord injured patients within $72 \mathrm{~h}$ of injury was designed for elective use in NASCIS 3. The purpose of NASCIS 3 was to evaluate the efficacy and safety of methylprednisolone infused over $48 \mathrm{~h}$ (48 MP), or tirilazad mesylate administered every $6 \mathrm{~h}$ for $48 \mathrm{~h}(48 \mathrm{TM})$, with $24 \mathrm{~h}$ of methylprednisolone (24 MP). ${ }^{9}$

The objective of this analysis is to evaluate the prognostic value of MR imaging for randomized patients in NASCIS 3, by determining whether the degree of neurological recovery at 6 weeks, as noted by changes in motor, pin, and touch responses can be predicted by MR imaging taken within $72 \mathrm{~h}$ of injury.

\section{Methods}

The data for this study are from the 499 patients randomized in NASCIS 3 between December 1991 and September 1995. MR imaging studies were performed electively on 282 patients during the acute care period; however, only 191 patients whose MR imaging was done within $72 \mathrm{~h}$ of injury are included in this study as that is the time period most likely to represent the initial injury. MR imaging included TI-weighted images with echo-delay time of $20-25 \mathrm{~ms}$ and pulse repetition time (TR) of $400-$ $800 \mathrm{~ms}$. T2-weighted images were done with a TE of $80 \mathrm{~ms}$ and TR of 2000 and TE 20 with TR 2000. Images were done in sagittal and axial planes with $3-5 \mathrm{~mm}$ slices through the area of interest with surface coils.

Questions to be completed on the NASCIS MRI form were: time and data of injury, time and date of MR imaging, whether signal intensity was consistent with acute hemorrhage, increased T2 signal intensity consistent with edema, signal changes consistent with contusion, a normal study, or other result. Conditions were noted as present or absent. The category of 'other' included such information as stenosis, cord compression, or bulging disks. Investigators were not asked to provide any technical information on the MR imaging unit, length or location of the cord lesion, or other details of MR imaging.

In some centers, results of MR imaging were transcribed from the patient's MRI report onto the appropriate NASCIS forms by study neurosurgical nurses. In other centers, MRI results were read directly from the MRI image itself onto the NASCIS forms by the neurosurgeon associated with NASCIS 3 .

Neurological examinations were performed by designated NASCIS personnel blind to which drug protocol the patient had been randomized. Patients were evaluated neurologically according to response to pin prick, light touch, and motor function at the time of admission to the study, and at preselected times in the year following the injury. Twenty-nine spinal cord segments, tested bilaterally for response to pin prick and light touch, were scored at $1=$ absent, $2=$ dysfunctional and $3=$ normal. Sensory scores ranged from a total score of 87 indicating normal response to a score of 29 indicating no response in any segment. Responses for the right side were summed, unless some cord segments could not be tested, in which case the left side was used. Motor function was determined by measuring bilaterally fourteen muscle roots, with $0=$ no contraction, $1=$ flicker/trace of contraction, $2=$ active movement without antigravity, $3=$ active movement with antigravity, $4=$ active movement against resistance, and normal $=5$. The responses for the right side were summed and ranged from 0 to 70 . Neurological change at 6 weeks after injury was determined by subtracting the motor and sensory score at the time of admission from the respective score at 6 weeks.

For purposes of this analysis we have only considered spinal cord surgery performed within $72 \mathrm{~h}$ of injury, to correspond with the timing of the first MR imaging.

When we reviewed the distribution of extent of injury among those whose MR imaging was in the 'other' category, 41 of these patients were noted as having a complete cord injury, and 31 were listed as incomplete. Twelve of the 'other' also had hemorrhage in the canal.

Chi square tests for independence were used to analyze categorical data, and Student's $t$-test and analysis of variance to compare means of continuous data. Multiple logistic regression with complete vs incomplete spinal cord injury (with complete defined as no motor or sensory function below the level of the lesion) as the outcome variable was modeled separately for hemorrhage, contusion and edema, while statistically controlling for differences in baseline motor, pin and touch scores.

Linear regression models with change in motor, pin, and touch scores at 6 weeks as outcome variables were analyzed to determine whether neurological improvement could be predicted from results of the MRI. Neurological improvement was defined as an improvement in motor, pin and touch scores at 6 weeks after injury. Independent variables in the statistical models were hemorrhage, contusion, or edema as identified by MR imaging, the three drug protocols, two levels of spinal cord injury: complete, and incomplete, spinal cord surgery: yes or no, and neurological measurements of motor function, response to pin prick, and 
touch, obtained at time of hospital admission, as described above.

\section{Results}

Overall, evidence of hemorrhage is observed in $17.3 \%$ of MRI scans, contusion in $26.7 \%$ and edema in $40.8 \%$. Twelve per cent of the patients were noted as having evidence of more than one condition on their scans. There are no statistically significant differences between the presence or absence of hemorrhage, contusion or edema, and gender, ethnicity, or age of the patients (Table 1).

Patients with a complete cord injury on admission, as noted radiologically and by neurological examination, are significantly more likely to have indications of hemorrhage in the spinal cord than those classified as neurologically normal on motor function but with impaired sensation $(26.7 \%$ vs $3.4 \%)$, while hemorrhage was observed in $13.8 \%$ of those with incomplete lesions $(P=0.01)$. Although presence of contusion or edema is more common in complete cord injury, these differences are not statistically significant.

Patients with evidence of contusion are significantly more likely to have received bolus doses of steroids before randomization. Those with evidence of edema are significantly less likely to have been given steroids before study enrollment, while pre-randomization steroids are unrelated to the presence of hemorrhage. We found a significantly greater proportion of patients with contusion on MRI in the 24 MP protocol and a significantly greater proportion of patients with edema in the $48 \mathrm{MP}$ protocol. The distribution of patients with hemorrhage on MR imaging is similar in all three drug protocols.

Although there is a slight increase in the proportion of patients with contusion who had their MR imaging later than $9 \mathrm{~h}$ after injury (the median time for MRI), the differences in time elapsed from injury to MRI and diagnoses of hemorrhage, contusion or edema were not statistically significant. Nor are there significant differences in MR results between those who had surgery 3 days or less after injury and those who did not.

When considering neurological function on admission, patients whose MR imaging results indicate hemorrhage or contusion are significantly more likely to have lower motor, pin, and touch scores at the time of admission compared to those with no such indications (Table 2). However, the ER motor, pin, and touch responses were unrelated to the presence of edema.

Table 2 also shows the neurological recovery change scores at 6 weeks by the MRI results. Overall, motor function recovery was less in patients with positive indications of hemorrhage, contusion and edema, but those differences are small and not statistically significant. The sensory scores showed no clear pattern of recovery by MRI indication.
We conducted multiple logistic regression with complete cord injury diagnosed neurologically on admission as the outcome variable of interest. We found the crude odds of a complete cord injury significantly increased if there was evidence of cord hemorrhage (OR 2.88, 95\% $\mathrm{Cl} 1.32,6.23)$, but less so if there was contusion (OR $1.73,95 \% \mathrm{Cl} 0.90,3.31)$ or edema (OR $1.4895 \% \mathrm{Cl} 0.82,2.68)$. To see how much

Table 1 Proportion of patients with MRI indications of hemorrhage, contusion, and edema, according to selected patient characteristics National Acute Spinal Cord Injury Study 3

\begin{tabular}{|c|c|c|c|c|}
\hline Characteristics & $\mathrm{n}$ & $\begin{array}{c}\text { Positive } M \\
\text { Hemorrhage } \\
(\%)\end{array}$ & $\begin{array}{l}R \text { imaging } \\
\text { Contusion } \\
(\%)\end{array}$ & $\begin{array}{l}\text { results } \\
\text { Edema } \\
(\%)\end{array}$ \\
\hline \multicolumn{5}{|l|}{ Gender } \\
\hline Male & 162 & 16.7 & 27.8 & 42.0 \\
\hline Female & 29 & $\begin{array}{c}20.7 \\
P=0.60\end{array}$ & $\begin{array}{c}20.7 \\
P=0.43\end{array}$ & $\begin{array}{c}34.5 \\
P=0.45\end{array}$ \\
\hline \multicolumn{5}{|l|}{ Ethnicity } \\
\hline African American & 24 & 25.0 & 16.7 & 50.0 \\
\hline White & 149 & 16.1 & 28.8 & 36.9 \\
\hline Hispanic & 9 & 0.0 & 33.3 & 77.8 \\
\hline Asian & 40 & 40.0 & 0.0 & 20.0 \\
\hline Native American & 1 & 0.0 & 100.0 & 100.0 \\
\hline Other & 3 & $\begin{array}{c}33.3 \\
P=0.35\end{array}$ & $\begin{array}{c}0.0 \\
P=0.19\end{array}$ & $\begin{array}{c}66.7 \\
P=0.07\end{array}$ \\
\hline \multicolumn{5}{|l|}{ Age (years) } \\
\hline $14-22$ & 49 & 18.4 & 22.4 & 40.8 \\
\hline $23-34$ & 54 & 11.1 & 27.8 & 35.2 \\
\hline $35-50$ & 41 & 19.5 & 31.7 & 48.8 \\
\hline Over 50 & 47 & $\begin{array}{c}21.3 \\
P=0.54\end{array}$ & $\begin{array}{c}25.5 \\
P=0.79\end{array}$ & $\begin{array}{c}40.4 \\
P=0.62\end{array}$ \\
\hline \multicolumn{5}{|l|}{ Extent of injury* } \\
\hline Complete & 75 & 26.7 & 33.3 & 46.7 \\
\hline Incomplete & 87 & 13.8 & 23.0 & 41.4 \\
\hline Normal & 29 & $\begin{array}{c}3.4 \\
P=0.01\end{array}$ & $\begin{array}{c}20.7 \\
P=0.24\end{array}$ & $\begin{array}{c}24.1 \\
P=0.11\end{array}$ \\
\hline \multicolumn{5}{|c|}{ Steroids pre-randomization } \\
\hline Yes & 139 & 16.5 & 31.6 & 36.0 \\
\hline No & 52 & $\begin{array}{c}19.2 \\
P=0.67\end{array}$ & $\begin{array}{c}13.5 \\
P=0.01\end{array}$ & $\begin{array}{c}53.8 \\
P=0.02\end{array}$ \\
\hline \multicolumn{5}{|l|}{ Study protocol } \\
\hline $48 \mathrm{~h}$ MP & 60 & 13.3 & 23.3 & 51.7 \\
\hline 48 h TM 64 & & 19.4 & 17.9 & 41.8 \\
\hline $24 \mathrm{~h} \mathrm{MP}$ & 67 & $\begin{array}{c}18.7 \\
P=0.62\end{array}$ & $\begin{array}{c}39.1 \\
P=0.02\end{array}$ & $\begin{array}{c}29.7 \\
P=0.04\end{array}$ \\
\hline \multicolumn{5}{|l|}{ Hours injury to MRI } \\
\hline Up to $9 \mathrm{~h}$ & 99 & 13.4 & 21.6 & 36.4 \\
\hline Over $9 \mathrm{~h}$ & 92 & $\begin{array}{c}21.7 \\
P=0.12\end{array}$ & $\begin{array}{c}32.6 \\
P=0.07\end{array}$ & $\begin{array}{c}45.6 \\
P=0.19\end{array}$ \\
\hline $\begin{array}{l}\text { Spinal surgery } \\
\qquad=3 \text { days } \\
>3 \text { days/none }\end{array}$ & $\begin{array}{r}86 \\
105\end{array}$ & $\begin{array}{c}21.1 \\
13.3 \\
P=0.11\end{array}$ & $\begin{array}{c}29.1 \\
24.8 \\
P=0.50\end{array}$ & $\begin{array}{c}46.5 \\
36.2 \\
P=0.15\end{array}$ \\
\hline
\end{tabular}

*Extent of injury determined radiologically and by neurological examination at time of admission. Normal function refers to motor scores but all these patients had impaired sensation. $P$-values refer to comparisons within each MRI indication 
MRI contributed to the diagnosis of complete injury independent of the neurological examination, we adjusted for neurological scores at admission in the logistic regression. The adjusted odds ratio (AOR) for a complete cord injury with $\mathrm{MR}$ imaging results indicating hemorrhage, contusion, or edema, were reduced: hemorrhage, AOR 1.43, $(95 \% \mathrm{Cl} 0.55$, 3.73); contusion, AOR $1.02(95 \% \mathrm{Cl} 0.42,2.45)$; and edema, AOR $1.33(95 \% \mathrm{Cl} 0.63,2.45)$. All confidence levels include one, reflecting a lack of statistical significance.

Table 2 Neurological scores at admission and 6 week change scores, by MRI results indicating hemorrhage, contusion, and edema National Acute Spinal Cord Injury Study $3^{\mathrm{a}, \mathrm{b}}$

\begin{tabular}{|c|c|c|c|}
\hline & \multicolumn{3}{|c|}{$M R$ imaging results } \\
\hline & $\begin{array}{c}\text { Hemorrhage } \\
(\%)\end{array}$ & $\begin{array}{l}\text { Contusion } \\
(\%)\end{array}$ & $\begin{array}{c}\text { Edema } \\
(\%)\end{array}$ \\
\hline \multicolumn{4}{|l|}{ Motor score in ER } \\
\hline MRI result positive & 24.3 & 26.9 & 31.0 \\
\hline SD & 19.1 & 22.6 & 21.6 \\
\hline MRI result negative & 34.3 & 34.6 & 33.6 \\
\hline \multirow[t]{2}{*}{ SD } & 22.2 & 21.5 & 22.3 \\
\hline & $P=0.02$ & $P=0.03$ & $P=0.43$ \\
\hline \multicolumn{4}{|l|}{ Pinprick score in ER } \\
\hline MRI result positive & 51.6 & 56.0 & 58.5 \\
\hline SD & 14.7 & 18.9 & 19.0 \\
\hline MRI result negative & 62.5 & 62.3 & 62.1 \\
\hline \multirow[t]{2}{*}{$\mathrm{SD}$} & 18.9 & 18.3 & 18.4 \\
\hline & $P=0.002$ & $P=0.04$ & $P=0.19$ \\
\hline \multicolumn{4}{|l|}{ Touch score in ER } \\
\hline MRI result positive & 52.0 & 57.6 & 60.9 \\
\hline SD & 14.7 & 19.5 & 19.6 \\
\hline MRI result negative & 64.9 & 64.5 & 63.9 \\
\hline \multirow[t]{2}{*}{$\mathrm{SD}$} & 19.0 & 18.5 & 18.5 \\
\hline & $P=0.0003$ & $P=0.03$ & $P=0.29$ \\
\hline \multicolumn{4}{|c|}{ Change in motor score at 6 weeks } \\
\hline MRI result positive & 7.4 & 10.1 & 8.2 \\
\hline SD & 10.9 & 13.8 & 15.2 \\
\hline MRI result negative & 11.3 & 10.7 & 12.2 \\
\hline \multirow[t]{2}{*}{ SD } & 16.3 & 16.2 & 15.6 \\
\hline & $P=0.10$ & $P=0.81$ & $P=0.09$ \\
\hline \multicolumn{4}{|c|}{ Change in pin score at 6 weeks } \\
\hline MRI result positive & 10.2 & 7.5 & 8.6 \\
\hline SD & 14.5 & 14.5 & 13.7 \\
\hline MRI result negative & 8.9 & 9.7 & 9.5 \\
\hline \multirow[t]{2}{*}{ SD } & 14.7 & 14.7 & 15.3 \\
\hline & $P=0.63$ & $P=0.38$ & $P=0.71$ \\
\hline \multicolumn{4}{|c|}{ Change in touch score at 6 weeks } \\
\hline MRI result positive & 10.7 & 9.3 & 7.2 \\
\hline SD & 14.1 & 15.0 & 13.0 \\
\hline MRI result negative & 8.1 & 8.3 & 9.5 \\
\hline \multirow[t]{2}{*}{ SD } & 14.7 & 14.2 & 15.5 \\
\hline & $P=0.38$ & $P=0.70$ & $P=0.30$ \\
\hline
\end{tabular}

${ }^{\mathrm{a}}$ Motor scores range from 0 (no contraction on any muscle) to 70 (all normal responses). Pin and touch scores range from 29 (all responses absent) to 87 (all responses normal). ${ }^{\mathrm{b}} P_{-}$ values calculated from Student's $t$-test, and refer to comparisons within each MRI indication
Table 2 also shows the neurological recovery change scores at 6 weeks by MRI results. Overall, motor function recovery was less in patients with positive indications of hemorrhage, contusion and edema but those differences were small and not statistically significant. The sensory scores showed no clear pattern of recovery by MRI indication.

Table 3 shows the association of MRI diagnoses of hemorrhage, contusion, and edema on recovery in motor, pin prick, and touch neurological scores at 6 weeks while controlling for ER motor score, complete cord injury, surgery, and drug protocols. Although not statistically significant, hemorrhage in the cord is associated with some reduction in motor function $(-2.7$ points) at 6 weeks, but is unrelated to change in response to pin prick and touch. Cord contusion is unrelated to recovery of motor function, but has a negative association on recovery to pin prick sensation $(-3.35$ points); edema is associated with reductions in motor function $(-3.34)$ and light touch sensation $(-3.41)$, both of borderline statistical significance.

\section{Conclusion}

Before controlling for neurological assessment scores at the time of admission, the presence of hemorrhage on MR imaging appeared significantly likely to be predictive of a neurologically complete cord injury, while contusion and edema were unrelated to severity of injury. However, when admission neurological scores were added to logistic regression models, none of the adjusted odds ratios for MRI parameters significantly predicted a complete cord injury. Thus, from the instrumentation used in the study, MRI results do not appear to add much to the diagnosis of a neurologically complete injury beyond the diagnosis

Table 3 Magnetic resonance imaging and neurological change at 6 weeks after injury: results of multiple regression analyses National Acute Spinal Cord Injury Study 3

\begin{tabular}{lccc}
\hline & \multicolumn{3}{c}{ Neurological improvement scores } \\
& Motor & at 6 weeks & Pin \\
Positive MRI result & function & prick & touch \\
\hline Hemorrhage & -2.70 & -0.63 & -0.93 \\
& $(0.29)$ & $(0.79)$ & $(0.69)$ \\
Contusion & -0.36 & -3.35 & -1.38 \\
& $(0.87)$ & $(0.09)$ & $(0.49)$ \\
Edema & -3.34 & -2.46 & -3.41 \\
& $(0.06)$ & $(0.16)$ & $(0.05)$ \\
\hline
\end{tabular}

Each estimate adjusted for: complete/incomplete cord injury assessed in ER; surgery within $72 \mathrm{~h}$ of injury or no surgery; baseline (emergency room) neurologic scores for each parameter of change: motor function, pin prick, and light touch, and study drug protocol. $P$-values for estimates are given in parentheses. Neurologic improvement scores are the neurologic score in the emergency room subtracted from the 6 week score 
made on the basis of the neurological examination performed on admission.

When considering the prognostic value of MR imaging, evidence of edema in the spinal cord was a better predictor of worse recovery of motor function and touch 6 weeks after injury, than was hemorrhage or contusion, after adjusting for all neurologic baseline parameters in the regression analyses. Neither hemorrhage nor contusion was predictive of recovery of motor function, pin prick or light touch at 6 weeks. We have restricted the outcome studied to a 6 week follow-up which is the time when recovery from spinal injury can be first observed without the influence of initial spinal shock. The 6 week recovery rates are highly predictive of neurological recovery observed at 6 months and 1 year. 9

This study builds on existing MR imaging research in acute spinal cord injury. Earlier studies determined the accuracy of MR imaging to show pathology of the injured cord, typically at the time of admission. While there are methodological and design differences between our study and the others we have cited, our study is in general agreement that the presence of hemorrhage in the spinal cord is indicative of a severe cord injury. $^{2-7}$ Except for the Flanders et $a l^{7}$ study, prior studies are based on small numbers, ${ }^{2,3,5}$ or technical descriptions of MR scanners and their accuracy in depicting cord pathology. 3,4 The early studies $^{2-4}$ focused on the correlation of MR imaging results with neurological prognosis, and reported that the presence of hemorrhage, edema, or cord compression on MRI was an indicator of poor neurological recovery.

Flanders et $a,^{7}$ using motor scales from the American Spinal Injury Association (ASIA) and extensive multivariate analyses, found that information about hemorrhage and proximal-distal range of edema around the lesion as observed from MR imaging, increased the prognostic ability to predict clinical outcome $16 \%$ to $33 \%$ more than just clinical scores alone. While our study also examines the predictive capabilities of MR imaging, we differ from the Flanders et $a l^{7}$ study in several ways: the size of the study sample, the inclusion of drug protocols in the analyses, the inclusion of sensory assessment as well as motor function, and the use of sensitive NASCIS neurological scoring (which is almost identical to the ASIA scales) to measure each spinal cord segment and muscle root. One weakness of our study is the lack of MR imaging results for the entire 499 NASCIS patients. This relatively restricted study sample explains the imbalance across MR imaging results. Like any study of technological screening, our analysis only reflects the sensitivity of MR imaging at time of study. New advances in imaging may lead to very different conclusions.

The present analyses find that MR imaging does not add much to the diagnosis of neurologic function at the time of injury when compared with assessment by more traditional measures of neurologic function.
However, MR imaging results for hemorrhage and contusion are both well correlated with the traditional standard neurological examination. Evidence of edema by MR imaging, however, does appear to confer some additional prognostic value for a worse outcome in motor function and light touch sensation at 6 weeks, beyond the predictive value of the baseline neurologic status.

MR imaging provides valuable diagnostic information on the degree of damage to bone and soft tissue of the spine at the time of injury. As MR imaging techniques continue to be refined, they are likely to offer stronger predictions of neurological recovery. Future studies examining the role of MR imaging in predicting outcome need to control for drug/steroid therapy, surgical intervention and other maneuvers which may modify the prognostic value of imaging.

\section{Acknowledgements}

The authors wish to thank Camilla Lindan, MD at Kaiser Permanente in San Francisco and Joseph Piepmeier, M.D. at Yale University School of Medicine for their assistance on this paper. Thanks also to the National Institute of Health (NS15078) for their support of the authors with this study.

\section{References}

1 Anderson BJ, Stringer WA. Imaging after spinal injury. In Narayan RK, Wilberger JE, Povlishock JT (eds). Neurotrauma. McGraw Hill Health Professions Division, New York, 1995, p. 1150.

2 Yamishita $\mathrm{Y}$ et al. Acute spinal cord injury: magnetic resonance imaging correlated with myelopathy. Br J Radiol 1991; 64: 201 209.

3 Bouderant FJ et al. Acute spinal cord injury. A study using physical examination and magnetic resonance imaging. Spine 1990; 15: $161-168$.

4 Flanders AE et al. Acute cervical spine trauma: correlation of MR findings with degree of neurological deficit. Radiology 1990; 177: $25-33$.

5 Marciello MA et al. Magnetic resonance imaging related to neurologic outcome in cervical spinal cord injury. Arch Phys Med Rehabil 1993; 74: 940 - 946.

6 Schaefer DM, Flanders AE, Osterholm JL, Northrup BE. Prognostic significance of magnetic resonance imaging in the acute phase of cervical spine injury. J Neurosurg 1992; 76: 218 223.

7 Flanders AE et al. Forecasting motor recovery after cervical spinal cord injury: value of MR imaging. Radiology 1996; 201: $649-655$.

8 Bracken MB et al. A randomized controlled trial of methylprednisolone or naloxone in the treatment of acute spinal cord injury: results of the Second National Acute Spinal Cord Study. $N$ Engl J Med 1990; 322: $1405-1411$.

9 Bracken MB et al. Administration of methylprednisolone for 24 or 48 hours, or tirilazad mesylate, in the treatment of acute spinal cord injury: results of the third National Acute Spinal Cord injury randomized controlled trial. JAMA 1997; 277: 1597 - 1604

10 Bracken MB et al. Methylprednisolone for 24 or 48 hours or tirilazad mesylate for 48 hours after acute spinal cord injury: 1 year follow-up. J Neurosurg 1998; 89: 699-706. 